\title{
ASYMPTOTICS OF SOLUTIONS OF THE WAVE EQUATION ON DE SITTER-SCHWARZSCHILD SPACE
}

\author{
RICHARD MELROSE, ANTÔNIO SÁ BARRETO, AND ANDRÁS VASY
}

\begin{abstract}
Solutions to the wave equation on de Sitter-Schwarzschild space with smooth initial data on a Cauchy surface are shown to decay exponentially to a constant at temporal infinity, with corresponding uniform decay on the appropriately compactified space.
\end{abstract}

\section{INTRODUCTION}

In this paper we describe the asymptotics of solutions to the wave equation on de Sitter-Schwarzschild space. The static model for the latter is $M=\mathbb{R}_{t} \times X$, $X=\left(r_{\mathrm{bh}}, r_{\mathrm{dS}}\right)_{r} \times \mathbb{S}_{\omega}^{2}$ with the Lorentzian metric

$$
g=\mu d t^{2}-\mu^{-1} d r^{2}-r^{2} d \omega^{2},
$$

where

$$
\mu=1-\frac{2 m}{r}-\frac{\Lambda r^{2}}{3}
$$

with $\Lambda$ and $m$ suitable positive constants, $0<9 m^{2} \Lambda<1, r_{\mathrm{bh}}, r_{\mathrm{dS}}$ the two positive roots of $\mu$ and $d \omega^{2}$ the standard metric on $\mathbb{S}^{2}$. We also consider the compactification of $X$ to

$$
\bar{X}=\left[r_{\mathrm{bh}}, r_{\mathrm{dS}}\right]_{r} \times \mathbb{S}_{\omega}^{2} .
$$

Then $\mu$ is a defining function for $\partial \bar{X}$ since it vanishes simply at $r_{\mathrm{bh}}, r_{\mathrm{dS}}$, i.e. $2 \beta=$ $\frac{d \mu}{d r} \neq 0$ at $r=r_{\mathrm{bh}}, r_{\mathrm{dS}}$. Moreover, in what follows we will sometimes consider

$$
\alpha=\mu^{\frac{1}{2}}
$$

as a boundary defining function for a different compactification of $X$. This amounts to changing the $\mathcal{C}^{\infty}$ structure of $\bar{X}$ by adjoining $\alpha$ as a smooth function. We denote the new manifold by $X_{\frac{1}{2}}$.

The d'Alembertian with respect to (1.1) is

$$
\square=\alpha^{-2}\left(D_{t}^{2}-\alpha^{2} r^{-2} D_{r}\left(r^{2} \alpha^{2} D_{r}\right)-\alpha^{2} r^{-2} \Delta_{\omega}\right),
$$

where $\Delta_{\omega}$ is the Laplacian on $\mathbb{S}^{2}$. We shall consider solutions to $\square u=0$ on $M$.

Regarding space-time as a product, up to the conformal factor $\alpha^{2}$, is in fact misleading in several ways - in particular, solutions to the wave equation do not have simple asymptotic behavior on this space. Starting from the stationary description

Date: 12 November, 2008.

2000 Mathematics Subject Classification. 35L05, 35P25, 83C57, 83C30.

The authors gratefully acknowledge financial support for this project, the first from the National Science Foundation under grant DMS-0408993, the second under grant DMS-0500788, and the third under grant DMS-0201092 and DMS-0801226; they are also grateful for the environment at the Mathematical Sciences Research Institute, Berkeley, where this paper was completed. 
of the metric, it is natural to first compactify the time line exponentially to an interval $[0,1]_{T}$. This can be done using a diffeomorphism $T: \mathbb{R} \rightarrow(0,1)$ with derivative $T^{\prime}<0$. Set

$$
T_{+}=T_{\lambda,+}=e^{-2 \lambda t} \text { in } t>C,
$$

with $\lambda$ to be determined and let

$$
T=T_{+} \text {in } t>C .
$$

Similarly set

$$
T_{-}=T_{\lambda,-}=e^{2 \lambda t}, T=1-T_{-} \text {in } t<-C .
$$

Near infinity $T$ depends on the free parameter $\lambda$. The boundary hypersurface $T_{+}=0$ (i.e. $T=0)$ in

$$
[0,1]_{T} \times \bar{X}
$$

is called here the future temporal face, $T_{-}=0$ the past temporal face, while $r=r_{\mathrm{bh}}$ and $r=r_{\mathrm{dS}}$ are the black hole, resp. de Sitter, infinity, or together spatial infinity.

In fact, it turns out that we need to use different values of $\lambda$ at the two ends, $\lambda_{\mathrm{bh}}$ and $\lambda_{\mathrm{dS}}$. This is discussed in more detail in the next section. There are product decompositions near these boundaries

$$
[0,1]_{T} \times\left[r_{\mathrm{bh}}, r_{\mathrm{bh}}+\delta\right) \times \mathbb{S}^{2},[0,1]_{T} \times\left(r_{\mathrm{dS}}-\delta, r_{\mathrm{dS}}\right] \times \mathbb{S}^{2}
$$

If $\delta$ is so large that these overlap, the transition function is not smooth but rather is given by taking positive powers of the defining function of the future temporal face, so the resulting space should really be thought of having a polyhomogeneous conormal (but not smooth) structure in the sense of differentiability up to the temporal faces. In particular, there is no globally preferred boundary defining function for the temporal face, rather such a function is only determined up to positive powers and multiplication by positive factors. Thus, there is no fully natural 'unit' of decay but we consider powers of $e^{-t}$, resp. $e^{t}$, in a neighborhood of the future and past temporal faces, respectively.

It turns out that there are two resolutions of this compactified space which play a useful role in describing asymptotics. The first arises by blowing up the corners

$$
\{0\} \times\left\{r_{\mathrm{bh}}\right\} \times \mathbb{S}^{2},\{0\} \times\left\{r_{\mathrm{dS}}\right\} \times \mathbb{S}^{2},\{1\} \times\left\{r_{\mathrm{bh}}\right\} \times \mathbb{S}^{2},\{1\} \times\left\{r_{\mathrm{dS}}\right\} \times \mathbb{S}^{2},
$$

where the blow-up is understood to be the standard spherical blow-up when locally the future temporal face is defined by $T_{\lambda_{\mathrm{dS}},+}$, resp. $T_{\lambda_{\mathrm{bh}},+}$ at the de Sitter and black hole ends. The resulting space is denoted $\bar{M}$. The lift of the temporal and spatial faces retain their names, while the new front faces are called the scattering faces. This is closely related to to the Penrose compactification, where however the temporal faces are compressed.

Thus, a neighborhood of the lift of $\{0\} \times\left\{r_{\mathrm{bh}}\right\} \times \mathbb{S}^{2}$ is diffeomorphic to

$$
[0, \epsilon)_{\rho} \times\left[r_{\mathrm{bh}}, r_{\mathrm{bh}}+\delta\right) \times \mathbb{S}_{\omega}^{2}, \rho=\rho_{\mathrm{bh},+}=T_{\lambda_{\mathrm{bh}},+} / \mu .
$$

Similarly, a neighborhood of $\{0\} \times\left\{r_{\mathrm{dS}}\right\} \times \mathbb{S}^{2}$ is diffeomorphic to

$$
[0, \epsilon)_{\rho} \times\left(r_{\mathrm{dS}}-\delta, r_{\mathrm{dS}}\right] \times \mathbb{S}_{\omega}^{2}, \rho=\rho_{\mathrm{dS},+}=T_{\lambda_{\mathrm{dS}},+} / \mu
$$

If $\delta>0$ is large enough, these cover a neighborhood of the future temporal face $\mathrm{tf}_{+}$, given by the lift of $T=0$. Thus a neighborhood of the interior of $\mathrm{tf}_{+}$, is 
polyhomogeneous-diffeomorphic to an open subset of

$$
\begin{aligned}
& {[0, \epsilon)_{x} \times\left(r_{\mathrm{bh}}, r_{\mathrm{dS}}\right) \times \mathbb{S}_{\omega}^{2},} \\
& x=\rho_{\mathrm{bh},+}^{1 /\left(2 \lambda_{\mathrm{bh},+}\right)} \text { for } r \text { near } r_{\mathrm{bh}}, x=\rho_{\mathrm{dS},+}^{1 /\left(2 \lambda_{\mathrm{dS},+}\right)} \text { for } r \text { near } r_{\mathrm{dS}},
\end{aligned}
$$

where we let the preferred defining function (up to taking positive multiples) of $\mathrm{tf}_{+}$ be $x=e^{-t}$ in the interior of $\mathrm{tf}_{+}$, hence $x=\rho_{\mathrm{bh},+}^{1 /\left(2 \lambda_{\mathrm{bh},+}\right)}$ at the black hole boundary of $\mathrm{tf}_{+}$. This means, in particular, that a neighborhood of $\mathrm{tf}_{+}$is polyhomogeneous diffeomorphic to

$$
[0, \epsilon)_{x} \times\left[r_{\mathrm{bh}}, r_{\mathrm{dS}}\right] \times \mathbb{S}_{\omega}^{2} .
$$

If $\mu$ is replaced by $\alpha$ as the defining function of the boundary of $X$, i.e. $\bar{X}$ and $T_{+}$are replaced by $\bar{X}_{1 / 2}$ and $T_{+}^{1 / 2}$ (and analogously in the past) the resulting space is denoted $\bar{M}_{1 / 2}$. Thus $\bar{M}_{1 / 2}$ is the square-root blow up of $\bar{M}$, where the square root of the defining function of every boundary hypersurface has been appended to the smooth structure. Here $\mathrm{tf}_{+}$is naturally diffeomorphic to $\bar{X}$ in $\bar{M}$, and to $\bar{X}_{1 / 2}$ in $\bar{M}_{1 / 2}$. Both $\bar{M}$ and $\bar{M}_{1 / 2}$ have polyhomogeneous conormal structures at $\mathrm{tf}_{+}$and $\mathrm{tf}_{-}$; we let the preferred defining function (up to taking positive multiples) of $\mathrm{tf}_{+}$ be $x=e^{-t}$ in the interior of $\mathrm{tf}_{+}$, hence $x=\rho^{1 /(2 \lambda)}$ at $\partial \mathrm{tf}_{+}$.

$\overline{\mathbb{R}} \times \bar{X}$

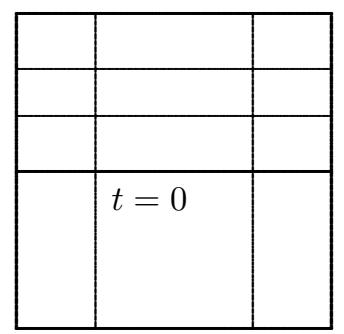

$\bar{M}$

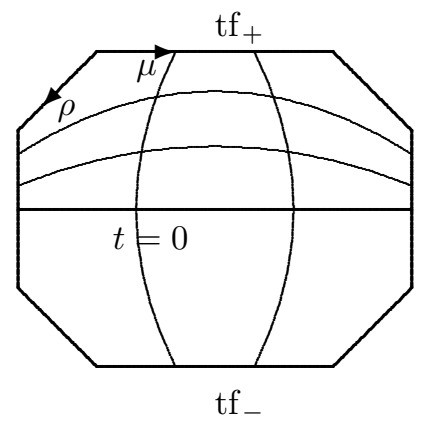

Figure 1. On the left, the space-time product compactification of de Sitter-Schwarzschild space is shown (ignoring the product with $\mathbb{S}^{2}$ ), with the time and space coordinate lines indicated by thin lines. On the right, $\bar{M}$ is shown, with the time and space coordinates indicated by thin lines. These are no longer valid coordinates on $\bar{M}$. Valid coordinates near the top left corner are $\rho$ and $\mu$.

Solutions to the wave equation, when lifted to this space have simpler asymptotics than on the product compactification, (1.8). The first indication of this is that $g$ extends to be $\mathcal{C}^{\infty}$ and non-degenerate, up to the scattering faces, $\mu=0$, away from spatial infinity and uniformly up to the temporal face; the scattering faces are characteristic with respect to the metric. We can thus extend $\bar{M}$ across 
$\mu=0$ to a manifold $\tilde{M}$, by allowing $\mu$ to take negative values; then the scattering face becomes an interior characteristic hypersurface.

A further indication of the utility of this space can be seen from our main result which is stated in terms of

$$
\mathcal{A}_{\mathrm{tf}_{+}}^{m}(\bar{M}) \text {. }
$$

This consists of those functions which are $\mathcal{C}^{\infty}$ on $\bar{M}$ away from $\mathrm{tf}_{+}$, and are conormal at $\mathrm{tf}_{+}$, including smoothness up to the boundary of $\mathrm{tf}_{+}$. Such spaces are welldefined, even though the smooth structure on $\bar{M}$ is not; the conormal structure suffices. Thus, the elements of (1.13), are fixed by the condition that for any $k$ and smooth vector fields $V_{1}, \ldots, V_{k}$ on $\bar{M}$ which are tangent to $\mathrm{tf}_{+}$,

$$
V_{1} \ldots V_{k} v \in x^{m} L_{\mathrm{b}, \mathrm{tf}+}^{2}(\bar{M}),
$$

where $L_{\mathrm{b}, \mathrm{tf}_{+}}^{2}(\bar{M})$ is the $L^{2}$-space with respect a density $\nu_{\mathrm{b}}$ such that $x \nu_{\mathrm{b}}$ is smooth and strictly positive on $\bar{M}$. Such a density is well-defined up to a strictly positive polyhomogeneous multiple even under the operation of replacing $x$ by a positive power, although the weight $x^{m}$ is not. Thus, for all $m \in \mathbb{R}$,

$$
x^{m+\epsilon} \mathcal{C}^{\infty}(\bar{M}) \subset \mathcal{A}_{\mathrm{tf}_{+}}^{m}(\bar{M}) \subset x^{m} L^{\infty}(\bar{M}), \epsilon>0 .
$$

The main result on wave propagation is:

Theorem 1.1. Suppose $u \in \mathcal{C}^{\infty}(\bar{M})$ satisfies $\square u=0$ for $x \in(0,1)$, then there exists a constant $c$ and $\epsilon>0$ such that

$$
u-c \in \mathcal{A}_{\mathrm{tf}_{+}}^{\epsilon}(\bar{M})=x^{\epsilon} \mathcal{A}_{\mathrm{tf}_{+}}^{0}(\bar{M}) .
$$

Thus, $u$ has an asymptotic limit, which happens to be a constant, at $\mathrm{tf}_{+}$, uniformly on $\bar{X}$.

While we have concerned ourselves with the behavior of the metric at the corner, in regions where $\rho<C$ (i.e. near temporal infinity), it is worthwhile considering what happens where $\rho>C$, i.e. at spatial infinity. As we shall see, spatial infinity can be blown down, i.e. there is a manifold $\overline{\mathrm{M}}$ and a $\mathcal{C}^{\infty}$ map $\beta, \beta: \bar{M} \rightarrow \overline{\mathrm{M}}$ such that $\beta$ is a diffeomorphism away from spatial infinity, and such that $g$ lifts to a $\mathcal{C}^{\infty}$ Lorentz b-metric on $\bar{M}$, with tangent (i.e. b-) behavior at the temporal face, smooth at the other faces, with respect to which the non-temporal faces are characteristic. One valid coordinate system in a neighborhood of the image of a neighborhood of the black hole end of spatial infinity, disjoint from temporal infinity, is given by exponentiated versions of Eddington-Finkelstein coordinates. In our notation, this corresponds to

$$
s_{\mathrm{bh},+}=\alpha / T_{\lambda_{\mathrm{bh},+}}^{1 / 2}=\rho_{\mathrm{bh},+}^{-1 / 2}, s_{\mathrm{bh},-}=\alpha / T_{\lambda_{\mathrm{bh},-}}^{1 / 2}=\alpha T_{\lambda_{\mathrm{bh},+}}^{1 / 2}=\mu \rho_{\mathrm{bh},+}^{1 / 2}, \omega,
$$

where as usual $\omega$ denotes coordinates on $\mathbb{S}^{2}$. Here

$$
\mathcal{F}_{\text {bh, }+}=\left\{s_{\text {bh },-}=0\right\}
$$

is the characteristic surface given by $\mu=0$ in $T>0$ (i.e. the front face of the blow up of the corner), and

$$
\mathcal{F}_{\mathrm{bh},-}=\left\{s_{\mathrm{bh},+}=0\right\}
$$

is its negative time analogue. The change of coordinates $\left(\rho_{\mathrm{bh},+}, \mu\right) \mapsto\left(s_{\mathrm{bh},+}, s_{\mathrm{bh},-}\right)$ is a diffeomorphism from $(0, \infty) \times(0, \delta)$ onto its image, i.e. these coordinates are indeed compatible. As we show in the next section, the metric is $\mathcal{C}^{\infty}$ and nondegenerate on $\overline{\mathrm{M}}$, and the boundary faces $s_{\mathrm{bh},+}=0$ and $s_{\mathrm{bh},-}=0$ are characteristic. 
We can again extend $\overline{\mathrm{M}}$ to $\widetilde{\mathrm{M}}$, which has only two boundary faces (the two temporal ones) by allowing $s_{\mathrm{bh}, \pm}$, and analogously $s_{\mathrm{dS}, \pm}$, to take on negative values. Thus, $\overline{\mathrm{M}}$ has six boundary faces,

$$
\mathrm{tf}_{+}, \mathrm{tf}_{-}, \mathcal{F}_{\mathrm{bh}, \pm}, \mathcal{F}_{\mathrm{dS}, \pm}
$$

called the future and past temporal faces, and the future $(+)$ and past $(-)$ black hole and de Sitter scattering faces.
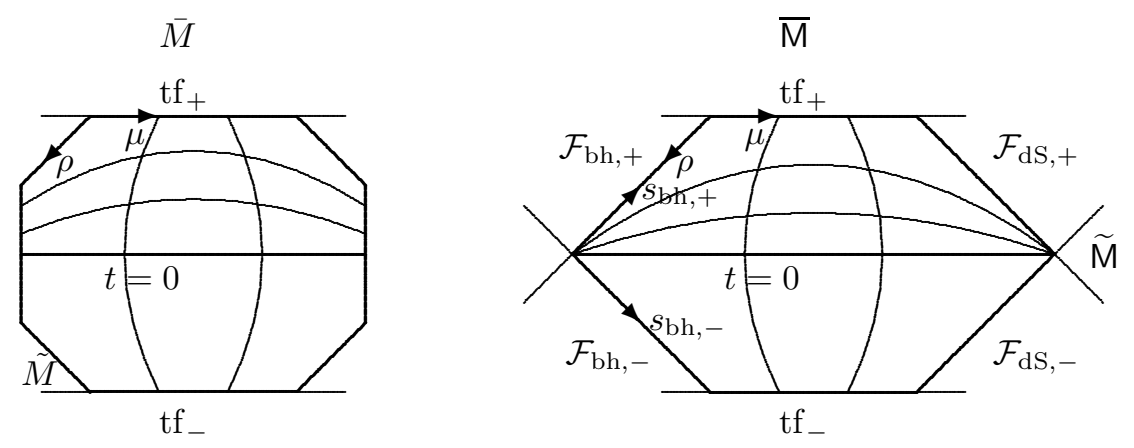

Figure 2. On the left, $\bar{M}$ is shown, while on the right its blowdown $\bar{M}$. The time and space coordinate lines corresponding to the product decomposition are indicated by thin lines in the interior. The temporal boundary hypersurfaces of $\bar{M}$ are continued by thin lines, as are the characteristic surfaces $\mathcal{F}_{\mathrm{bh}, \pm}$ and $\mathcal{F}_{\mathrm{dS}, \pm}$, to show that the Lorentz metric extends smoothly across $\mathcal{F}_{\mathrm{bh}, \pm}$ and $\mathcal{F}_{\mathrm{dS}, \pm}$ (but not across the temporal face!). The extended spaces are denoted by $\tilde{M}$ and $\widetilde{M}$. Valid coordinates near $\mathcal{F}_{\mathrm{bh},+} \cap \mathcal{F}_{\mathrm{bh},-}$ are (apart from the spherical coordinates) $s_{\mathrm{bh},+}$ and $s_{\mathrm{bh},-}$, as shown.

The following propagation result follows directly from the properties of this blowdown.

Proposition 1.2. If $u$ satisfies $\square u=0$ and has $\mathcal{C}^{\infty}$ Cauchy data on a space-like Cauchy surface $\Sigma \subset \widetilde{\mathrm{M}} \cap\{t \geq 0\}$, for example $\Sigma=\{t=0\}$ (i.e. $s_{\mathrm{bh},+}=s_{\mathrm{bh},-}$ ), then $u \in \mathcal{C}^{\infty}\left(\overline{\mathrm{M}}^{\circ}\right)$.

Combining Proposition 1.2 and Theorem [1.1 leads to the main result of this paper:

Theorem 1.3. If $u$ satisfies $\square u=0$ and has $\mathcal{C}^{\infty}$ Cauchy data on a space-like Cauchy surface $\Sigma \subset \widetilde{M} \cap\{t \geq 0\}$ then there exists a constant $c$ and $\epsilon>0$ such that

$$
u-c \in \mathcal{A}_{\mathrm{tf}_{+}}^{\epsilon}(\overline{\mathrm{M}})=x^{\epsilon} \mathcal{A}_{\mathrm{tf}_{+}}^{0}(\overline{\mathrm{M}})
$$

near the future temporal face, $\mathrm{tf}_{+}$. 
Remark 1.4. Our methods extend further, for example to Cauchy data at $t=0$ which are conormal at $\partial \bar{X}$, of growth $s_{\mathrm{bh},+}^{m}, m>-2$, at the boundary. Standard hyperbolic propagation gives the same behavior at $\mathcal{F}_{\mathrm{bh},+}$ and $\mathcal{F}_{\mathrm{dS},+}$ in $\rho<C$, and then the resolvent estimates for the 'spatial Laplacian' $\Delta_{X}$ (described below) apply to yield the same asymptotic term but with convergence in the appropriate conormal space, including conormality with respect to $\mathcal{F}_{\mathrm{bh},+}$ and $\mathcal{F}_{\mathrm{dS},+}$.

Remark 1.5. Dafermos and Rodnianski [4] have proved, by rather different methods, a similar result with an arbitrary logarithmic decay rate, i.e. an analogue of

$$
u-c \in(\log \rho)^{-N} \mathcal{A}_{\mathrm{tf}_{+}}^{0}(\overline{\mathrm{M}})
$$

for every $N$. In terms of our approach, such logarithmic convergence follows from polynomial bounds on the resolvent of $\Delta_{X}$ at the real axis, rather than in a strip for the analytic continuation; such estimates are much easier to obtain, as is explained below.

As already indicated, by looking at the appropriate compactification, one only needs to study the asymptotics near $\mathrm{tf}_{+}$in $\bar{M}$ (or equivalently, $\overline{\mathrm{M}}$ ). We do this by taking the Mellin transform of the wave equation and using high-energy resolvent estimates for a 'Laplacian' $\Delta_{X}$ on $\bar{X}$. A conjugated version of this operator is asymptotically hyperbolic, hence fits into the framework of Mazzeo and the first author [7, which in particular shows the existence of an analytic continuation for the resolvent

$$
R(\sigma)=\left(\Delta_{X}-\sigma^{2}\right)^{-1}, \operatorname{Im} \sigma<0 .
$$

Here we also need high-energy estimates for $R(\sigma)$.

The operator $\Delta_{X}$ has been studied by the second author and Zworski in 9 , where it is shown (using the spherical symmetry to reduce to a one-dimensional problem and applying complex scaling) that the resolvent admits an analytic continuation, from the 'physical half plane', with only one pole, at 0 , in $\operatorname{Im} \sigma<\epsilon$, for $\epsilon$ sufficiently small. Bony and Hafner in [1] extend and refine this result to derive polynomial bounds on the cutoff resolvent, $\chi R(\sigma) \chi, \chi \in \mathcal{C}_{\mathrm{c}}^{\infty}(X)$, as $|\sigma| \rightarrow \infty$ in the strip $|\operatorname{Im} \sigma|<\epsilon$. This implies that, for initial data in $\mathcal{C}_{\mathrm{c}}^{\infty}(X)$, the local energy, i.e. the energy in a fixed compact set in space, decays to the energy corresponding to the 0 -resonance. In our terminology this amounts to studying the behavior of the solution near a compact subset of the interior of $\mathrm{tf}_{+}$. Our extension of their result is both to allow more general initial data, not necessarily of compact support, and to study the asymptotics uniformly up to the boundary at temporal infinity. This requires resolvent estimates on slightly weighted $L^{2}$-spaces, which were obtained by the authors in 8 together with the use of the geometric compactification $\bar{M}$ (or $\overline{\mathrm{M}})$. For this to succeed, it is essential that the resolvent only be applied to 'errors' which intersect $\partial \overline{\mathrm{M}}$ in the interior of $\mathcal{F}_{\mathrm{bh},+}$ and $\mathcal{F}_{\mathrm{dS},+}$. This turns out to be a major gain since the analytic continuation of the resolvent (even arbitrarily close to the real axis) cannot be applied directly to the initial data. Thus essential use is made of the fact that once the solution has been propagated to the scattering faces, the error terms have more decay.

It is then relatively clear, as remarked above, that if one only knew polynomial growth estimates for the limiting resolvent at the real axis (rather than in a strip), one could still obtain the same asymptotics, but with error that is only super-logarithmically decaying. This observation may be of use in other settings where such polynomial bounds are relatively easy to obtain from estimates for the 
cutoff resolvent, as in [1], or analogous semiclassical propagation estimates at the trapped set, by pasting with well-known high energy resolvent estimates localized near infinity. This has been studied particularly by Cardoso and Vodev [3], using the method of Bruneau and Petkov [2, Section 3].

This paper is structured as follows. In Section 2 both the compactifications and the underlying geometry are discussed in more detail. The 'spatial Laplacian' and relevant resolvent estimates are recalled in Section 3 and in Section 4 the main result is proved using the Mellin transform.

\section{Geometry}

In this section the various compactifications of de Sitter-Schwarzschild space are studied after an initial examination of the simpler case of de Sitter space.

2.1. De Sitter space. We start with the extreme case of de Sitter space, corresponding to $m=0$ in (1.1) and (1.2), to see what the 'correct' compactification of $M$ should be. However, rather than starting from the static model, consider this as a Lorentzian symmetric space. De Sitter space is given by the hyperboloid

$$
z_{1}^{2}+\ldots+z_{n}^{2}=z_{n+1}^{2}+1 \text { in } \mathbb{R}^{n+1}
$$

equipped with the pull-back of the Minkowski metric

$$
d z_{n+1}^{2}-d z_{1}^{2}-\ldots-d z_{n}^{2}
$$

Introducing polar coordinates $(R, \theta)$ in $\left(z_{1}, \ldots, z_{n}\right)$, so

$$
R=\sqrt{z_{1}^{2}+\ldots+z_{n}^{2}}=\sqrt{1+z_{n+1}^{2}}, \theta=R^{-1}\left(z_{1}, \ldots, z_{n}\right) \in \mathbb{S}^{n-1}, \tau=z_{n+1},
$$

the hyperboloid can be identified with $\mathbb{R}_{\tau} \times \mathbb{S}_{\theta}^{n-1}$ with the Lorentzian metric

$$
\frac{d \tau^{2}}{\tau^{2}+1}-\left(\tau^{2}+1\right) d \theta^{2}
$$

where $d \theta^{2}$ is the standard Riemannian metric on the sphere. For $\tau>1$, set $x=\tau^{-1}$, so the metric becomes

$$
\frac{\left(1+x^{2}\right)^{-1} d x^{2}-\left(1+x^{2}\right) d \theta^{2}}{x^{2}} .
$$

An analogous formula holds for $\tau<-1$, so compactifying the real line to an interval $[0,1]_{T}$, with $T=x=\tau^{-1}$ for $x<\frac{1}{4}$ (i.e. $\tau>4$ ), say, and $T=1-|\tau|^{-1}, \tau<-4$, gives a compactification, $\widehat{M}$, of de Sitter space on which the metric is conformal to a non-degenerate Lorentz metric. There is natural generalization, to asymptotically de Sitter-like spaces $\widehat{\mathrm{M}}$, which are diffeomorphic to compactifications $[0,1]_{T} \times Y$ of $\mathbb{R}_{\tau} \times Y$, where $Y$ is a compact manifold without boundary, and $\widehat{M}$ is equipped with a Lorentz metric on its interior which is conformal to a Lorentz metric smooth up to the boundary. These space-times are Lorentzian analogues of the muchstudied conformally compact (Riemannian) spaces. On this class of space-times the solutions of the Klein-Gordon equation were analyzed by the third author in [10], and were shown to have simple asymptotics analogous to those for eigenfunctions on conformally compact manifolds. 
Theorem. ([10, Theorem 1.1.]) Set $s_{ \pm}(\lambda)=\frac{n-1}{2} \pm \sqrt{\frac{(n-1)^{2}}{4}-\lambda}$. If $s_{+}(\lambda)-s_{-}(\lambda) \notin$ $\mathbb{N}$, any solution $u$ of the Cauchy problem for $\square-\lambda$ with $\mathcal{C}^{\infty}$ initial data at $\tau=0$ is of the form

$$
u=x^{s_{+}(\lambda)} v_{+}+x^{s_{-}(\lambda)} v_{-}, v_{ \pm} \in \mathcal{C}^{\infty}(\widehat{\mathrm{M}}) .
$$

If $s_{+}(\lambda)-s_{-}(\lambda)$ is an integer, the same conclusion holds if $v_{-} \in \mathcal{C}^{\infty}(\widehat{\mathrm{M}})$ is replaced by $v_{-}=\mathcal{C}^{\infty}(\widehat{\mathrm{M}})+x^{s_{+}(\lambda)-s_{-}(\lambda)} \log x \mathcal{C}^{\infty}(\widehat{\mathrm{M}})$.
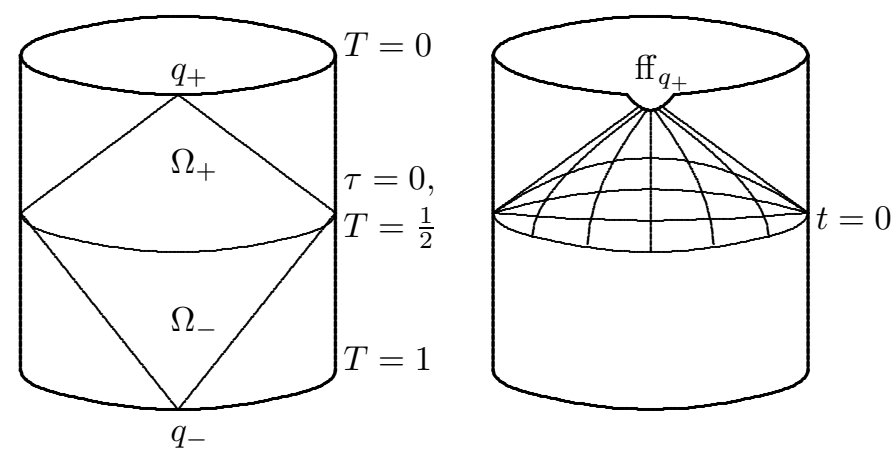

Figure 3. On the left, the compactification of de Sitter space with the backward light cone from $q_{+}=(1,0,0,0)$ and forward light cone from $q_{-}=(-1,0,0,0)$ are shown. $\Omega_{+}$, resp. $\Omega_{-}$, denotes the intersection of these light cones with $t>0$, resp. $t<0$. On the right, the blow up of de Sitter space at $q_{+}$is shown. The interior of the light cone inside the front face $\mathrm{ff}_{q_{+}}$can be identified with the spatial part of the static model of de Sitter space. The spatial and temporal coordinate lines for the static model are also shown.

The simple structure of the de Sitter metric (and to some extent the asymptotically de Sitter-like metrics) can be hidden by blowing up certain submanifolds of $\widehat{M}$. In particular, the static model of de Sitter space arises by singling out a point on $\mathbb{S}_{\theta}^{n-1}$, e.g. $q_{0}=(1,0, \ldots, 0) \in \mathbb{S}^{n-1} \subset \mathbb{R}^{n}$. Note that $\left(\theta_{2}, \ldots, \theta_{n}\right) \in \mathbb{R}^{n-1}$ are local coordinates on $\mathbb{S}^{n-1}$ near $q_{0}$. Now consider the intersection of the backward light cone from $q_{0}$ considered as a point $q_{+}$at future infinity, i.e. where $T=0$, and the forward light cone from $q_{0}$ considered as a point $q_{-}$at past infinity, i.e. where $T=1$. These intersect the equator $T=1 / 2$ (here $\tau=0$ ) in the same set, and together form a 'diamond', $\hat{\Omega}$, with a conic singularity at $q_{+}$and $q_{-}$. Explicitly $\hat{\Omega}$ is given by $z_{2}^{2}+\ldots+z_{n}^{2} \leq 1$ inside the hyperboloid. If $q_{+}, q_{-}$are blown up, as well as the corner $\partial \Omega \cap\{\tau=0\}$, i.e. where the light cones intersect $\tau=0$ in $\hat{\Omega}$, we obtain a manifold $\bar{M}$, which can be blown down to (i.e. is a blow up of) the space-time product $[0,1] \times \overline{\mathbb{B}^{n-1}}$, with $\mathbb{B}^{n-1}=\left\{Z \in \mathbb{R}^{n-1}:|Z|<1\right\}$ on which the Lorentz metric has a time-translation invariant warped product form. Namely, first considering the interior $\Omega$ of $\hat{\Omega}$ we introduce the global (in $\Omega$ ) standard static 
coordinates $(t, Z)$, given by (with the expressions involving $x$ valid near $T=0$ )

$$
\begin{aligned}
\mathbb{B}^{n-1} \ni Z & =\left(z_{2}, \ldots, z_{n}\right)=x^{-1} \sqrt{1+x^{2}}\left(\theta_{2}, \ldots, \theta_{n}\right), \\
\sinh t & =\frac{z_{n+1}}{\sqrt{z_{1}^{2}-z_{n+1}^{2}}}=\left(x^{2}-\left(1+x^{2}\right)\left(\theta_{2}^{2}+\ldots+\theta_{n}^{2}\right)\right)^{-1 / 2},
\end{aligned}
$$

It is convenient to rewrite these as well in terms of polar coordinates in $Z$ (valid away from $Z=0)$ :

$$
\begin{gathered}
r=\sqrt{z_{2}^{2}+\ldots+z_{n}^{2}}=\sqrt{1+z_{n+1}^{2}-z_{1}^{2}}=x^{-1} \sqrt{1+x^{2}} \sqrt{\theta_{2}^{2}+\ldots+\theta_{n}^{2}} \\
\sinh t=\frac{z_{n+1}}{\sqrt{z_{1}^{2}-z_{n+1}^{2}}}=\left(x^{2}-\left(1+x^{2}\right)\left(\theta_{2}^{2}+\ldots+\theta_{n}^{2}\right)\right)^{-1 / 2}=x^{-1}\left(1-r^{2}\right)^{-1 / 2}, \\
\omega=r^{-1}\left(z_{2}, \ldots, z_{n}\right)=\left(\theta_{2}^{2}+\ldots+\theta_{n}^{2}\right)^{-1 / 2}\left(\theta_{2}, \ldots, \theta_{n}\right) \in \mathbb{S}^{n-2} .
\end{gathered}
$$

In these coordinates the metric becomes

$$
\left(1-r^{2}\right) d t^{2}-\left(1-r^{2}\right)^{-1} d r^{2}-r^{2} d \omega^{2},
$$

which is a special case of the de Sitter-Schwarzschild metrics with $m=0$ and $\Lambda=3$.

Lemma 2.1. The lift of $\hat{\Omega}$ to the blow up $\left[\widehat{\mathrm{M}} ; q_{+}, q_{-}\right]$is a $\mathcal{C}^{\infty}$ manifold with corners, $\bar{\Omega}$. Moreover, $[\bar{\Omega}, \partial \Omega \cap\{\tau=0\}]$ is naturally diffeomorphic to the $\mathcal{C}^{\infty}$ manifold with corners obtained from $\left[[0,1] \times \overline{\mathbb{B}^{n-1}} ;\{0\} \times \partial \mathbb{B}^{n-1} ;\{1\} \times \partial \mathbb{B}^{n-1}\right]$ by adding the square root of the defining function of the lift of $\{0\} \times \overline{\mathbb{B}^{n-1}}$ and $\{1\} \times \overline{\mathbb{B}^{n-1}}$ to the $\mathcal{C}^{\infty}$ structure.

Remark 2.2. This lemma states that from the stationary point of view, the 'right' compactification near the top face arises by blowing up the corner $\partial \overline{\mathbb{R}} \times \partial \overline{\mathbb{B}^{n-1}}$, although the resulting space is actually more complicated than needed, since the original boundary hypersurfaces of the stationary space can be blown down to obtain a subset of $\widehat{M}$.

The fact that this approach gives $\rho=x^{2}$ as the defining function of the temporal face, rather than $x$ (hence necessitating adding the square root of $\rho$ to the smooth structure), corresponds to the fact that, in the sense of Guillarmou [5], $\widehat{M}$ is actually even.

Proof. Coordinates on the blow up of $\widehat{\mathrm{M}}$ near the lift of $q_{+}$are given by

$$
x, \Theta_{j}=\frac{\sqrt{1+x^{2}}}{x} \theta_{j}, j=2, \ldots, n ;
$$

these are all bounded in the region of validity of the coordinates, with the light cone given by $\sum_{j=2}^{n} \Theta_{j}^{2}=1$ (which is why the factor $\sqrt{1+x^{2}}$ was introduced), so the lift $\bar{\Omega}$ of $\hat{\Omega}$ is $\sum_{j=2}^{n} \Theta_{j}^{2} \leq 1$. As $\sum_{j=2}^{n} \Theta_{j}^{2}-1$ has a non-vanishing differential where it vanishes, this shows that $\bar{\Omega}$ is a $\mathcal{C}^{\infty}$ manifold with corners. Near the light cone, $\partial \bar{\Omega}$ one can introduce polar coordinates in $\Theta$, and use

$$
x, r=\left(\sum_{j=2}^{N} \Theta_{j}^{2}\right)^{1 / 2},\left(\omega_{2}, \ldots, \omega_{n}\right)=r^{-1}\left(\Theta_{2}, \ldots, \Theta_{n}\right) \in \mathbb{S}^{n-1}
$$


as local coordinates. On the other hand, blowing up the corner of $[0,1]_{T} \times \overline{\mathbb{B}^{n-1}}$, where $T=e^{-2 t}$ for $t>4$, say, which is equivalent to $(\sinh t)^{-2}$ there, gives coordinates near the lift of $T=0$ :

$$
r, \rho=(\sinh t)^{-2} /\left(1-r^{2}\right)=x^{2}, \omega .
$$

Thus, one almost has a diffeomorphism between the two coordinate charts, hence locally between the manifolds, except that in the blow up of $[0,1]_{T} \times \overline{\mathbb{B}^{n-1}}$, the defining function of the temporal face is the square of the defining function of the temporal face arising from the blow up of $\widehat{\mathrm{M}}$. This is remedied by adding the square root of the defining function of the lift of $\{0\} \times \overline{\mathbb{B}^{n-1}}$ and $\{1\} \times \overline{\mathbb{B}^{n-1}}$, i.e. of $x^{2}$, to the smooth structure, thus proving the lemma.

It is worthwhile comparing the de Sitter space wave asymptotics, which is

$$
u=x^{n-1} v_{+}+v_{-}, v_{+} \in \mathcal{C}^{\infty}(\widehat{\mathrm{M}}), v_{-} \in \mathcal{C}^{\infty}(\widehat{\mathrm{M}})+x^{n-1}(\log x) \mathcal{C}^{\infty}(\widehat{\mathrm{M}}),
$$

with our main result. The fact that the coefficients in the de Sitter expansion are $\mathcal{C}^{\infty}$ on $\widehat{M}$ means that on $\bar{M}$, the leading terms are constant. Thus, the de Sitter result implies (and is much stronger than) the statement that $u$ decays to a constant on $\bar{M}$ at an exponential rate.

2.2. Blow-up of the space-time product. We now turn to the compactification of de Sitter-Schwarzschild space. It turns out that while this cannot be embedded into a space as simple as $\widehat{M}$ the final setting is not much more complicated. In terms of de Sitter space, the difference is that while spatial infinity in $[0,1]_{T} \times \overline{\mathbb{B}^{3}}$, blown up at the corner, can always be blown down, the same is not true for temporal infinity.

In fact, the 'black hole end' $r=r_{\text {bh }}$ resembles the 'de Sitter end' quite closely, which motivates the construction in the de Sitter-Schwarzschild setting. There is a simpler construction, depending on the choice of a constant $\lambda>0$, which does not quite work because of some incompatibility between the two ends (which whilst very similar, are not quantitatively the same). With $\mu$ as in (1.2), compactify $M$ by compactifying $\mathbb{R}$ into $[0,1]_{T}$ as in (1.5)-(1.7), so

$$
T_{+}=T_{\lambda,+}=e^{-2 \lambda t} \text { in } t>c,
$$

and compactify $\left(r_{\mathrm{bh}}, r_{\mathrm{dS}}\right)$ as $\left[r_{\mathrm{bh}}, r_{\mathrm{dS}}\right]$, to obtain

$$
[0,1]_{T} \times\left[r_{\mathrm{bh}}, r_{\mathrm{dS}}\right] \times \mathbb{S}^{2}=[0,1]_{T} \times \bar{X} .
$$

Then blowing up the corners

$$
\{0\} \times\left\{r_{\mathrm{bh}}\right\} \times \mathbb{S}^{2},\{0\} \times\left\{r_{\mathrm{dS}}\right\} \times \mathbb{S}^{2}
$$

(and analogously at $T=1$, i.e. $T_{-}=0$ ), gives a space denoted $\bar{M}$. Thus, a neighborhood $U=U_{\lambda,+}$ of the 'temporal face' $T_{+}=0$ is diffeomorphic to

$$
[0, \epsilon)_{\rho} \times\left[r_{\mathrm{bh}}, r_{\mathrm{dS}}\right] \times \mathbb{S}_{\omega}^{2}, \rho=T_{\lambda,+} / \mu .
$$

In the interior of the temporal face, where $\mu>0$, this is in turn diffeomorphic to an open subset of

$$
[0, \epsilon)_{T} \times\left(r_{\mathrm{bh}}, r_{\mathrm{dS}}\right) \times \mathbb{S}_{\omega}^{2}
$$


If the same construction is performed, but using the smooth structure on the the compactification of $X$ given by $\alpha=\mu^{1 / 2}$, i.e. $\bar{X}_{1 / 2}$, and

$$
\tilde{T}_{\lambda,+}=T_{\lambda,+}^{1 / 2}=e^{-\lambda t}, t>c
$$

then a neighborhood $U$ of $\mathrm{tf}_{+}$as above is diffeomorphic to

$$
[0, \epsilon)_{\tilde{\rho}} \times\left[r_{\mathrm{bh}}, r_{\mathrm{dS}}\right]_{1 / 2} \times \mathbb{S}_{\omega}^{2}, \tilde{\rho}=\tilde{T}_{\lambda,+} / \alpha,
$$

where $\left[r_{\mathrm{bh}}, r_{\mathrm{dS}}\right]_{1 / 2}$ denotes that $\alpha$ has been added to the smooth structure (or equivalently $\left(r-r_{\mathrm{bh}}\right)^{1 / 2}$ and $\left(r_{\mathrm{dS}}-r\right)^{1 / 2}$ have been added to the smooth structure). The distinction between (2.1) and (2.3) is the same as between $[0,1]_{T} \times \bar{X}$ and $[0,1]_{\tilde{T}} \times \bar{X}_{1 / 2}$ (where $\tilde{T}$ is defined analogously to $T$ ), namely the square roots of the defining functions of all boundary hypersurfaces have been added to the smooth structure. We denote the resulting space by $\bar{M}_{1 / 2}$.

The subtlety is that the de Sitter and black hole ends need different values of $\lambda$. So what we actually need is to paste together $U_{\lambda_{\mathrm{bh}},+} \cap\left\{r<r_{2}\right\}$ and $U_{\lambda_{\mathrm{dS}},+} \cap\left\{r>r_{1}\right\}$ for some $r_{1}, r_{2}, r_{\mathrm{bh}}<r_{1}<r_{2}<r_{\mathrm{dS}}$ where $\lambda_{\mathrm{bh}}$ and $\lambda_{\mathrm{dS}}$ are chosen in a way that reflects the local geometry neat the two ends. The transition map in the overlap, where $r \in\left(r_{1}, r_{2}\right)$, is given by

$$
\left(\rho_{\mathrm{bh},+}, r, \omega\right) \mapsto\left(\rho_{\mathrm{dS},+}, r, \omega\right), \rho_{\mathrm{dS},+}=\mu^{\lambda_{\mathrm{dS}} / \lambda_{\mathrm{bh}}-1} \rho_{\mathrm{bh},+}^{\lambda_{\mathrm{dS}} / \lambda_{\mathrm{bh}}} .
$$

In the overlap, where $\mu \neq 0$ so $\mu \mapsto \mu^{\lambda_{\mathrm{dS}} / \lambda_{\mathrm{bh}}-1}$ is smooth, this is a polyhomogeneousdiffeomorphism, in the sense that it is polyhomogeneous in the local defining functions of the lift of $T=0$ (namely $\rho_{\mathrm{bh},+}$ and $\rho_{\mathrm{dS},+}$ ). In particular, the front faces of the blow-ups have well defined boundary defining functions, namely $\mu$, up to multiplying by a $\mathcal{C}^{\infty}$ non-vanishing function, so we consider the resulting space a polyhomogeneous manifold with corners, where the 'polyhomogeneous' (as opposed to $\mathcal{C}^{\infty}$ ) faces are only the future and past temporal faces, $\mathrm{tf}_{+}$and $\mathrm{tf}_{-}$. There is also an analogous construction for $\bar{M}_{1 / 2}$.

As indicated already, we also want a preferred defining function (up to taking positive multiples) $x$ of $\mathrm{tf}_{+}$in order to measure the rate of decay at the temporal faces; this should be polyhomogoneous-equivalent to the local defining functions $\rho_{\mathrm{bh},+}$ and $\rho_{\mathrm{dS},+}$. We take this to be of the form $x=f(r) e^{-t}, f>0$ smooth for $r \in\left(r_{\mathrm{bh}}, r_{\mathrm{dS}}\right)$. Comparison with (1.9)-(1.10) shows that we need to take $f(r)=$ $\mu^{-1 /\left(2 \lambda_{\mathrm{bh}}\right)}$ for $r$ near $r_{\mathrm{bh}}, f(r)=\mu^{-1 /\left(2 \lambda_{\mathrm{dS}}\right)}$ for $r$ near $r_{\mathrm{dS}}$, hence $x=\rho_{\mathrm{bh},+}^{1 /\left(2 \lambda_{\mathrm{bh}}\right)}$ near the black hole boundary of $\mathrm{tf}_{+}$, and $x=\rho_{\mathrm{bh},+}^{1 /\left(2 \lambda_{\mathrm{dS}}\right)}$ near the de Sitter boundary. Then, in particular, a neighborhood of $\mathrm{tf}_{+}$is polyhomogeneous diffeomorphic to

$$
[0, \epsilon)_{x} \times\left[r_{\mathrm{bh}}, r_{\mathrm{dS}}\right] \times \mathbb{S}_{\omega}^{2} .
$$

We still need to determine the values $\lambda$ at the two ends. Writing $T=T_{+}=T_{\lambda,+}$, the dual metric (which is the principal symbol of the wave operator, $\square$ ) has the form

$$
G=4 \alpha^{-2} \lambda^{2} T^{2} \partial_{T}^{2}-\alpha^{2} \partial_{r}^{2}-r^{-2} \partial_{\omega}^{2}
$$

in the original product compactification, with $\partial_{r}=\frac{d \mu}{d r} \partial_{\mu}=2 \beta \partial_{\mu}$. The change of variables from $r$ to $\mu$ is smooth and non-degenerate, i.e. $2 \beta=d \mu / d r \neq 0$ for $\mu$ close to 0 , i.e. $r$ close to $r_{\mathrm{bh}}$ or $r_{\mathrm{dS}}$. Note that $\beta>0$ for $r$ near $r_{\mathrm{bh}}, \beta<0$ for $r$ near $r_{\mathrm{dS}}$ since $\mu>0$ for $r \in\left(r_{\mathrm{bh}}, r_{\mathrm{dS}}\right)$.

After blow-up, in the coordinates $(\rho, \mu, \omega)$,

$$
G=4 \mu^{-1} \lambda^{2} \rho^{2} \partial_{\rho}^{2}-4 \mu \beta^{2}\left(\partial_{\mu}-\mu^{-1} \rho \partial_{\rho}\right)^{2}-r^{-2} \partial_{\omega}^{2} .
$$


Thus,

$$
G=\mu^{-1}\left(4 \lambda^{2} \rho^{2} \partial_{\rho}^{2}-4 \beta^{2}\left(\mu \partial_{\mu}-\rho \partial_{\rho}\right)^{2}\right)-r^{-2} \partial_{\omega}^{2} .
$$

If we set $\lambda=\beta\left(r_{\mathrm{bh}}\right)>0$ or $\lambda=-\beta\left(r_{\mathrm{dS}}\right)>0$ then 1 the $\rho^{2} \partial_{\rho}^{2}$ terms cancel, so locally near $r_{\mathrm{bh}}$

$$
G=4 \gamma \rho^{2} \partial_{\rho}^{2}+8 \beta^{2} \rho \partial_{\rho} \partial_{\mu}-4 \beta^{2} \mu \partial_{\mu}^{2}-r^{-2} \partial_{\omega}^{2}, \gamma=\mu^{-1}\left(\beta\left(r_{\mathrm{bh}}\right)^{2}-\beta^{2}\right),
$$

where $\gamma$ is $\mathcal{C}^{\infty}$ by Taylor's theorem, and there is a similar expansion at $r_{\mathrm{dS}}$. Thus the choice of $\lambda$ determines the compactification $\bar{M}$, and it is only at this point that the compactification has been specified. Note that this metric is a $\mathcal{C}^{\infty}$ Lorentzian b-metric on $[0, \epsilon)_{\rho} \times\left(r_{\mathrm{bh}}-\epsilon, r_{\mathrm{bh}}+\epsilon\right)_{r} \times \mathbb{S}_{\omega}^{2}$ (i.e. is non-degenerate as a quadratic form on the b-cotangent bundle), in particular it is $\mathcal{C}^{\infty}$ across $\mu=0$. Denoting this extension of $\bar{M}$ by $\tilde{M}$ (which is now non-compact); $g$ becomes a polyhomogeneous conormal Lorentz metric on $\tilde{M}$, smooth near $\mu=0$ (where there is a well-defined smooth structure). We write $\mathcal{F}$ for the set given by $\mu=0$, i.e. the boundary hypersurface of $\bar{M}$ that is no longer a boundary hypersurface of $\tilde{M}$.

For this metric $\mathcal{F}$ is characteristic, and one has the standard propagation of singularities in $\rho>0$. In particular, for $\mathcal{C}^{\infty}$ initial data the solution is smooth in $\rho>0$ across $\mu=0$. In fact, writing covectors as $\xi \frac{d \rho}{\rho}+\zeta d \mu+\sum \eta_{j} d \omega_{j}$, i.e. $(\rho, \mu, \omega, \xi, \zeta, \eta)$ are coordinates on ${ }^{\mathrm{b}} T^{*} \tilde{M}$, the dual metric, considered as a function on ${ }^{\mathrm{b}} T^{*} \tilde{M}$, is

$$
G=4 \gamma \xi^{2}+8 \beta^{2} \xi \zeta-4 \beta^{2} \mu \zeta^{2}-r^{-2}|\eta|^{2}
$$

so the Hamilton vector field of $G$ is

$$
\begin{aligned}
H_{G}= & 8\left(\gamma \xi+\beta^{2} \zeta\right) \rho \partial_{\rho}-8 \beta^{2}(\mu \zeta-\xi) \partial_{\mu} \\
& -\left(4 \frac{\partial \gamma}{\partial \mu} \xi^{2}+8 \beta \frac{\partial \beta}{\partial \mu}\left(2 \xi \zeta-\mu \zeta^{2}\right)-4 \beta^{2} \zeta^{2}-\frac{\partial r^{-2}}{\partial \mu}|\eta|^{2}\right) \partial_{\zeta}-r^{-2} H_{(\omega, \eta)},
\end{aligned}
$$

with $H_{(\omega, \eta)}$ denoting the Hamilton vector field of the standard metric on the sphere. The conormal bundle $N^{*}\{\mu=0\}$ is $\mu=0, \xi=0, \eta=0$, so at this set

$$
H_{G}=8 \beta^{2} \zeta \rho \partial_{\rho}+4 \beta^{2} \zeta^{2} \partial_{\zeta}
$$

and so is indeed tangent to $N^{*}\{\mu=0\}$, and it is non-radial off the zero section (where $\zeta \neq 0$ ) as long as $\rho \neq 0$.

At $\partial \mathcal{F}$, i.e. at $\rho=0$, however there are radial points over the conormal bundle of $\mathcal{F}$. Rather than dealing with them directly, which can be done in the spirit of [6], we reduce the problem to the study of the high energy behavior of the resolvent of the spatial Laplacian (which gives more, in fact), which was performed in 8 .

2.3. Blow down of spatial infinity. We now discuss the manifold with corners $\overline{\mathrm{M}}$, in which spatial infinity is blown down. A valid coordinate system near the image of the black hole end of spatial infinity, disjoint from temporal infinity, is given by

$$
s_{\mathrm{bh},+}=\alpha / T_{\lambda_{\mathrm{bh},+}}^{1 / 2}=\rho_{\mathrm{bh},+}^{-1 / 2}, s_{\mathrm{bh},-}=\alpha / T_{\lambda_{\mathrm{bh},-}}^{1 / 2}=\alpha T_{\lambda_{\mathrm{bh},+}}^{1 / 2}=\mu \rho_{\mathrm{bh},+}^{1 / 2}, \omega,
$$

where as usual $\omega$ denotes coordinates on $\mathbb{S}^{2}$. In these coordinates the dual metric is

$$
G=\gamma\left(s_{\mathrm{bh},+}^{2} \partial_{s_{\mathrm{bh},+}}^{2}+s_{\mathrm{bh},-}^{2} \partial_{s_{\mathrm{bh},-}}^{2}\right)-2\left(\beta\left(r_{\mathrm{bh}}\right)^{2}+\beta^{2}\right) \partial_{s_{\mathrm{bh},+}} \partial_{s_{\mathrm{bh},-}}-r^{-2} \partial_{\omega}^{2},
$$

\footnotetext{
${ }^{1}$ In terms of the 'spatial Laplacian', $\Delta_{X}$, described in Section $3-\beta\left(r_{\mathrm{bh}}\right)^{2}$ and $-\beta\left(r_{\mathrm{dS}}\right)^{2}$ are the asymptotic curvatures, hence they are natural quantities from that point of view as well.
} 
and the boundary faces $s_{\mathrm{bh},+}=0$ and $s_{\mathrm{bh},-}=0$ are characteristic. We can also extend $G$ to a smooth non-degenerate Lorentz metric on $\widetilde{\mathrm{M}}^{\circ}$. Recall that $\widetilde{\mathrm{M}}$ contains $\overline{\mathrm{M}}$ as a closed domain with corners, namely locally in $\widetilde{\mathrm{M}}$ we simply allow the four boundary defining functions $s_{\mathrm{bh}, \pm}, s_{\mathrm{dS}, \pm}$, to assume negative values (but we do not extend $\bar{M}$ across the temporal faces). This calculation shows the following important fact:

Lemma 2.3. The d'Alembertian $\square \in \operatorname{Diff}^{2}\left(\widetilde{\mathrm{M}}^{\circ}\right)$, and indeed $\square \in \operatorname{Diff}_{b}^{2}(\widetilde{\mathrm{M}})$. Moreover the scattering surfaces $\mathcal{F}_{\mathrm{bh}, \pm}=\left\{s_{\mathrm{bh}, \mp}=0\right\}$ and $\mathcal{F}_{\mathrm{dS}, \pm}=\left\{s_{\mathrm{dS}, \mp}=0\right\}$ are characteristic.

Proposition 1.2 is an immediate corollary of this lemma and standard hyperbolic propagation.

\section{Resolvent estimates}

Next consider the 'spatial Laplacian', resolvent estimates for which constitute one of the key ingredients in our analysis. From (1.4) with $T=e^{-t}$, it follows that

$$
\square=\alpha^{-2}\left(\left(T D_{T}\right)^{2}-\alpha^{2} r^{-2} D_{r} \alpha^{2} r^{2} D_{r}-\alpha^{2} r^{-2} \Delta_{\omega}\right) .
$$

Recall that not precisely $T$, but rather $T^{\lambda_{\mathrm{bh}}}$ and $T^{\lambda_{\mathrm{ds}}}$, were used above to construct the compactification.

By definition the spatial 'Laplacian' is

$$
\Delta_{X}=\alpha^{2} r^{-2} D_{r} \alpha^{2} r^{2} D_{r}+\alpha^{2} r^{-2} \Delta_{\omega}
$$

Near $\alpha=0$, where $\alpha$ can be used as a valid coordinate in place of $r$,

$$
\Delta_{X}=\beta r^{-2} \alpha D_{\alpha} \beta r^{2} \alpha D_{\alpha}+\alpha^{2} r^{-2} \Delta_{\omega} \in \operatorname{Diff}_{0}^{2}\left(\bar{X}_{1 / 2}\right) .
$$

This is not the Laplacian of a Riemannian metric on $X$; however it is very similar to one. It is of the form $d^{*} d$ with respect to the inner product on one-forms given by the fiber inner product with respect to the 'spatial part' $H=\alpha^{2} \partial_{r}^{2}+r^{-2} \partial_{\omega}^{2}$ of $G$ and density on $X$ given by $d h=\alpha^{-2} r^{2} d r d \omega$. In what follows we will also view $\Delta_{X}$ is a 0 -operator on $\bar{X}_{1 / 2}$, self-adjoint on

$$
L^{2}(X,|d h|),|d h|=\alpha^{-2} r^{2}|d r||d \omega|=\alpha^{-1}|\beta|^{-1} r^{2}|d \alpha||d \omega|,
$$

and we will use the techniques of [7] to study its resolvent. It is also useful to introduce the operator

$$
L=\alpha \Delta_{X} \alpha^{-1},
$$

which is self-adjoint on

$$
L^{2}\left(X, \alpha^{-2}|d h|\right)=\alpha L^{2}(X, d h), \alpha^{-2}|d h|=\alpha^{-3}|\beta|^{-1} r^{2}|d \alpha||d \omega| .
$$

Thus, this space is $L_{0}^{2}(X)$ as a Banach space, up to equivalence of norms.

Let $\tilde{\alpha}=\alpha^{1 / \lambda_{\mathrm{bh}}} \in \mathcal{C}^{\infty}(X), \tilde{\alpha}>0$, for $r$ near $r_{\mathrm{bh}}$ or $\tilde{\alpha}=\alpha^{1 / \lambda_{\mathrm{dS}}}$ for $r$ near $r_{\mathrm{dS}}$. The normal operators $N_{0, \mathrm{bh}}(L), N_{0, \mathrm{dS}}(L)$ of $L$ in $\operatorname{Diff}_{0}^{2}\left(\bar{X}_{1 / 2}\right)$ at $r=r_{\mathrm{bh}}$, resp. $r=r_{\mathrm{dS}}$, are

$$
N_{0, \mathrm{bh}}(L)=\lambda_{\mathrm{bh}}^{2} N_{0, \mathrm{bh}}\left(\Delta_{\mathbb{H}^{3}}\right), N_{0, \mathrm{dS}}(L)=\lambda_{\mathrm{dS}}^{2} N_{0, \mathrm{dS}}\left(\Delta_{\mathbb{H}^{3}}\right),
$$

where $\Delta_{\mathbb{H}^{3}}$ is the hyperbolic Laplacian, explaining the usefulness of this conjugation. In particular, it follows immediately from [7] (with improvements from [5]) that the resolvent

$$
\mathcal{R}(\sigma)=\left(L-\sigma^{2}\right)^{-1}, \text { on } L_{0}^{2}\left(\bar{X}_{1 / 2}\right), \operatorname{Im} \sigma<0,
$$


continues meromorphically to a strip $|\operatorname{Im} \sigma|<\epsilon$ as an operator between weighted $L^{2}$-spaces (as well as other spaces):

$$
\mathcal{R}(\sigma): \tilde{\alpha}^{\delta} L_{0}^{2}\left(\bar{X}_{1 / 2}\right) \rightarrow \tilde{\alpha}^{-\delta} L_{0}^{2}\left(\bar{X}_{1 / 2}\right), \delta>\epsilon ;
$$

we keep denoting the analytic continuation by $\mathcal{R}(\sigma)$. Thus,

$$
R(\sigma)=\left(\Delta_{X}-\sigma^{2}\right)^{-1}=\alpha^{-1} \mathcal{R}(\sigma) \alpha \text { on } L^{2}(X,|d h|), \operatorname{Im} \sigma<0,
$$

continues meromorphically to a strip $|\operatorname{Im} \sigma|<\epsilon$

$$
R(\sigma): \tilde{\alpha}^{\delta} L^{2}(X,|d h|) \rightarrow \tilde{\alpha}^{-\delta} L^{2}(X,|d h|), \delta>\epsilon .
$$

The result we need is proved in [8], giving polynomial bounds on the resolvent in a strip around the real axis.

Proposition 3.1. If $\epsilon>0$ is sufficiently small the only pole of the analytic continuation of the resolvent $R(\sigma)$ in $\operatorname{Im} \sigma<\epsilon$ is $\sigma=0$, which is simple, with residue given by a constant $\gamma$ and for each $k$ and $\delta>\epsilon$ there exist $m>0$ and $C>0$ and $M$ such that

$$
\left\|\tilde{\alpha}^{-i \sigma} R(\sigma)\right\|_{\mathcal{L}\left(\alpha^{-1} \tilde{\alpha}^{\delta} H_{0}^{m}\left(\bar{X}_{1 / 2}\right), C^{k}(\bar{X})\right)} \leq C|\sigma|^{M},
$$

for $|\sigma|>1, \operatorname{Im} \sigma<\epsilon$.

\section{Asymptotics For solutions of the WAVE EQUATION}

We now proceed to study the asymptotics of solutions of the wave equation at $\mathrm{tf}_{+}$.

Suppose $u$ is a solution of the wave equation, $\square u=0$, and $u$ is smooth on $\tilde{M}^{\circ}$. Energy estimates show that $u$ is necessarily tempered, in the sense that $u \in$ $\rho^{-s} L^{2}(\bar{M})$ for some $s>0$. This is shown directly below.

Let $\phi=\phi_{0}(\rho) \in \mathcal{C}^{\infty}(\bar{M})$ be a cutoff function, with $\rho \in \mathcal{C}_{\mathrm{c}}^{\infty}([0,1))$, identically 1 near 0 . If $u$ satisfies $\square u=0$ and $u$ is smooth in $\mu$ (i.e. across the side faces), then $\phi u$ is smooth in $\mu$, and

$$
\square(\phi u)=[\square, \phi] u=f
$$

where $f$ is also smooth in $\mu$, and vanishes in a neighborhood of the temporal face - since $[\square, \phi] \in \operatorname{Diff}_{\mathrm{b}}^{1}(\tilde{M})$, by Lemma 2.3 is supported away from the temporal face. Moreover, $v=\phi u$ is the unique solution of $\square v=f$ in $\bar{M}^{\circ}$ with $v=0$ for $\rho$ sufficiently large.

Now we wish to take the Mellin transform in $T=e^{-t}$, for functions supported in a neighborhood of the temporal face, namely in $U=\{\rho<1\}$. Such a neighborhood is equipped with a fibration $\bar{M} \rightarrow X$, extending the fibration $M \rightarrow X$ in the interior, and there is a natural density $|d t|=\frac{|d T|}{T}$ on the fibers. In coordinates $(\tilde{\rho}, \alpha, \omega)=\left(T^{\lambda_{\mathrm{bh}}} / \alpha, \alpha, \omega\right)$ valid near the temporal face boundary at $r=r_{\mathrm{bh}}$ this density takes the form $\frac{|d \tilde{\rho}|}{\lambda_{\mathrm{bh}} \tilde{\rho}}$. So, the Mellin transform can be taken with respect to this fibration and density. Thus the map $v \mapsto \hat{v}$ from functions supported near the temporal face to functions on $\Omega \times X, \Omega \subset \mathbb{C}$,

$$
\hat{v}(\sigma, z)=\int T^{i \sigma} v(T, z) \frac{|d T|}{T} .
$$

If $v$ is polynomially bounded in $T$, supported in $T \geq 0$, with values in a function space $\mathcal{H}$ in $z$, this transform gives an analytic function in a lower half plane (depending on the order of growth of $v$ ) with values in $\mathcal{H}$. 
In fact, writing the integral in coordinates valid near the boundary of the temporal face, $\tilde{\rho}=T^{\lambda_{\mathrm{bh}}} / \alpha$,

$$
\hat{v}(\sigma, \alpha, \omega)=\alpha^{i \sigma / \lambda_{\mathrm{bh}}} \lambda_{\mathrm{bh}}^{-1} \int \tilde{\rho}^{i \sigma / \lambda_{\mathrm{bh}}} v(\tilde{\rho}, \alpha, \omega) \frac{|d \tilde{\rho}|}{\tilde{\rho}} .
$$

The integral is then the Mellin transform of $v$ with respect to $\tilde{\rho}$ evaluated at $\sigma / \lambda_{\mathrm{bh}}$. Thus, if $v$ is smooth on $\tilde{M}^{\circ}$, supported in $\{\tilde{\rho} \in I\}, I \subset(0,1)$ compact, i.e. near but not at the temporal face, then $\hat{v}$ is in fact analytic in $\mathbb{C}$ with values in functions of the form $\tilde{\alpha}^{i \sigma} \mathcal{C}^{\infty}$, with $\mathcal{C}^{\infty}$ seminorms all bounded by $C_{k}\langle\sigma\rangle^{-k}, k$ arbitrary. If $v$ is just supported in $\tilde{\rho}<1$ and is conormal on $\tilde{M}$, then $\hat{v}$ is analytic in a lower half plane with values in functions of the form $\tilde{\alpha}^{i \sigma} \mathcal{C}^{\infty}$.

Assuming for the moment that $\phi u$ is polynomially bounded in $T, \square(\phi u)=f$ becomes $\hat{N}_{b}(\square) \widehat{\phi u}=\hat{f}$, where

$$
\hat{N}_{b}(\square)=\alpha^{-2}\left(\sigma^{2}-\Delta_{X}\right)=\alpha^{-2}\left(\sigma^{2}-\alpha^{2} r^{-2} D_{r} \alpha^{2} r^{2} D_{r}-\alpha^{2} r^{-2} \Delta_{\omega}\right),
$$

so

$$
\left(\sigma^{2}-\Delta_{X}\right) \widehat{\phi u}=\alpha^{2} \hat{f} .
$$

If $\phi u$ is polynomially bounded in $T$, then both $\hat{f}$ and $\widehat{\phi u}$ are analytic in $\operatorname{Im} \sigma<$ $-C$, and as $f$ is compactly supported in $\tilde{\rho}, \hat{f}$ is in entire analytic with values in functions of the form $\tilde{\alpha}^{i \sigma} \mathcal{C}^{\infty}$, with $\mathcal{C}^{\infty}$ seminorms all bounded by $C_{k}\langle\sigma\rangle^{-k}, k$ arbitrary. Thus,

$$
\widehat{\phi u}=R(\sigma)\left(\alpha^{2} \hat{f}\right), \operatorname{Im} \sigma<-C,
$$

and we recover $\phi u$ by taking the inverse Mellin transform.

We now return to arbitrary (not a priori polynomially bounded) $u, f=\square(\phi u)$, as above. Thus, $\hat{f}$ is analytic in all of $\mathbb{C}$, with values in functions of the form $\tilde{\alpha}^{i \sigma} \mathcal{C}^{\infty}$, with $\mathcal{C}^{\infty}$ seminorms all bounded by $C_{k}\langle\sigma\rangle^{-k}, k$ arbitrary. Now note that the inclusion

$$
\alpha^{1+s} L^{\infty}(X) \hookrightarrow L_{0}^{2}\left(\bar{X}_{1 / 2}\right)
$$

is continuous for every $s>0$. Thus, the inclusion

$$
\tilde{\alpha}^{i \sigma-\delta} \alpha^{3} \mathcal{C}^{\infty}\left(\bar{X}_{1 / 2}\right) \hookrightarrow H_{0}^{m}\left(\bar{X}_{1 / 2}\right)
$$

is continuous if

$$
\lambda(\epsilon+\delta)<2, \lambda=\max \left(\lambda_{\mathrm{bh}}, \lambda_{\mathrm{dS}}\right),
$$

and $\operatorname{Im} \sigma<\epsilon$, which is to say

$$
\tilde{\alpha}^{i \sigma} \alpha^{2} \mathcal{C}^{\infty}\left(\bar{X}_{1 / 2}\right) \hookrightarrow \alpha^{-1} \tilde{\alpha}^{\delta} H_{0}^{m}\left(\bar{X}_{1 / 2}\right)
$$

is continuous.

In particular, then

$$
\left\|\alpha^{2} \hat{f}\right\|_{\alpha^{-1} \tilde{\alpha}^{\delta} H_{0}^{m}\left(\bar{X}_{1 / 2}\right)} \leq C_{k}\langle\sigma\rangle^{-k}
$$

for all $k$ in $\operatorname{Im} \sigma<\epsilon<\delta$ (with new constants), $0<\epsilon<\delta$ sufficiently small. Proposition 3.1] shows that, for $\epsilon>0$ sufficiently small and for all $N$ and $k$,

$$
\left\|\tilde{\alpha}^{-i \sigma} R(\sigma)\left(\alpha^{2} \hat{f}\right)\right\|_{C^{N}(\bar{X})} \leq C_{k}|\sigma|^{-k}, \operatorname{Im} \sigma<\epsilon .
$$

The inverse Mellin transform of $w=R(\sigma)\left(\alpha^{2} \hat{f}\right)$ is

$$
\check{w}(T, z)=(2 \pi)^{-1} \int T^{-i \sigma} w(\sigma, z) d \sigma .
$$


Thus,

$$
\check{w}(\tilde{\rho}, \alpha, \omega)=(2 \pi)^{-1} \int \tilde{\rho}^{-i \sigma / \lambda_{\mathrm{bh}}} \alpha^{-i \sigma / \lambda_{\mathrm{bh}}} w(\sigma, \alpha, \omega) d \sigma .
$$

In view of (4.1) (in particular the analyticity of $\check{w}$ in the lower half plane with the stated estimates), $w=0$ for $T<0$ (as can be seen directly by shifting the contour to $\operatorname{Im} \sigma=-C$, using the off spectrum resolvent estimate $\|R(\sigma)\|_{\mathcal{L}\left(L^{2}(X,|d h|)\right)} \leq$ $\left|\operatorname{Im}\left(\sigma^{2}\right)\right|^{-1}$ and letting $\left.C \rightarrow+\infty\right)$. Since the unique solution of $\square v=f, v$ supported in $T \geq 0$, is $\phi u$, it follows that $\check{w}=\phi u$.

Shifting the contour for the inverse Mellin transform for $w$ to $\operatorname{Im} \sigma=\epsilon$ gives a residue term at 0 , and shows that

$$
\tilde{\rho}^{-\epsilon}(\phi u-v) \in L^{2}\left([0, \delta)_{\tilde{\rho}} ; \mathcal{C}^{\infty}(\bar{X})\right),
$$

where $v$ arises from the residue at 0 , hence is a constant function. Note that (at the cost of changing $\epsilon$ ) this is equivalent to the analogous statement with $\rho$ replaced by $\tilde{\rho}$. The derivatives with respect $t$ satisfy similar estimates. Hence, the same estimates hold for the conormal derivatives with respect to $\tilde{\rho}$ (or equivalently $\rho$ ). We thus deduce the leading part of the asymptotics of $u$ at the future temporal face, $\mathrm{tf}_{+}$.

This completes the proof of Theorem 1.1 The main result, Theorem 1.3 follows from the combination of this result with Proposition 1.2

\section{REFERENCES}

[1] J.-F. Bony and D. Hafner. Decay and non-decay of the local energy for the wave equation in the De Sitter - Schwarzschild metric. Preprint, arXiv:0706.0350, 2007.

[2] V. Bruneau and V. Petkov. Semiclassical resolvent estimates for trapping perturbations. Comm. Math. Phys., 213(2):413-432, 2000.

[3] F. Cardoso and G. Vodev. Uniform estimates of the resolvent of the Laplace-Beltrami operator on infinite volume Riemannian manifolds. II. Ann. Henri Poincaré, 3(4):673-691, 2002.

[4] M. Dafermos and I. Rodnianski. The wave equation on Schwarzschild-de Sitter space times. Preprint, arXiv:07092766, 2007.

[5] C. Guillarmou. Meromorphic properties of the resolvent on asymptotically hyperbolic manifolds. Duke Math. J., 129(1):1-37, 2005.

[6] A. Hassell, R. B. Melrose, and A. Vasy. Microlocal propagation near radial points and scattering for symbolic potentials of order zero. Analysis and PDE, To appear.

[7] R. Mazzeo and R. B. Melrose. Meromorphic extension of the resolvent on complete spaces with asymptotically constant negative curvature. J. Func. Anal., 75:260-310, 1987.

[8] R. B. Melrose, A. Sá Barreto, A. Vasy. Analytic continuation and semiclassical resolvent estimates on asymptotically hyperbolic spaces. In preparation.

[9] A. Sá Barreto and M. Zworski. Distribution of resonances for spherical black holes. Math. Res. Lett., 4(1):103-121, 1997.

[10] A. Vasy. The wave equation on asymptotically de Sitter-like spaces. Preprint, arxiv:math/07063669, 2007.

Department of Mathematics, Massachusetts Institute of Technology, Cambridge MA 02139-4307, U.S.A.

E-mail address: rbm@math.mit.edu

Department of Mathematics, Purdue University, West Lafayette, IN 47907-1395, U.S.A.

E-mail address: sabarre@math.purdue.edu

Department of Mathematics, Stanford University, Stanford, CA 94305-2125, U.S.A.

E-mail address: andras@math.stanford.edu 